\title{
THE INTERNET AS GOD: ANALYSING ASSESSMENT IN WEB 2.0
}

\author{
Kate Galloway* \\ Presentation at ALTA Conference \\ Sydney \\ 5-8 July 2009
}

\begin{abstract}
The advent of Web 2.0 is flagged as a revolution in technology having profound effects on global economic and social life. Web 2.0 harnesses the interconnectivity and interaction of the world wide web as a platform in itself. Education is not immune from this revolution, and has a lot to learn from the web 2.0 mantra 'don't fight the internet'. In the tertiary sector, while legal education in particular has arguably represented a more traditional approach to teaching and learning, many legal educators are integrating digital technologies into their teaching. This may represent a more contemporary approach to meeting our students' learning needs, however this paper argues that web 2.0 demands more of us than simply a more flexible or technologically current approach to our teaching and the tools we use to help our students learn. Instead, it requires a paradigm shift in how we conceptualise teaching and learning. This is illustrated through an analysis of the use of blogs in a first year assessment task.
\end{abstract}

\section{Introduction}

This paper is really a story about my experimentation with blogs in a first year law subject, undertaken in conjunction with colleagues with whom I have team taught.

While I will focus on the assessment angle of this story, I want to contextualise the use of this tool within the framework of web 2.0. This concept, web 2.0, in my view, represents the need for change in contemporary education from that which is integrated within an industrialised society, to that which provides a springboard for individual and collective creativity.

This creates a whole host of discussion points, but one key to understanding the paradigm shift in society wrought by the 'information revolution' and the concomitant need for development in the way we think about education, lies in the development and role of web2.0.

First then, I will run briefly through the concept of web2.0, highlighting the way in which I think it provides an imperative for us as educators to transform our practice. I will then provide a snapshot of one example of how a web2.0 tool, blogs, can be used within our practice to bring a contemporary flavour to our students' learning. Finally, I will highlight the aspects of subject design implicit in the selection of this medium for assessment, and the ongoing vital role of reflection in this process.

\footnotetext{
${ }^{*}$ Lecturer, School of Law, James Cook University.
} 


\section{What is Web2.0? or, 'Don't Fight the Internet'}

Eric Schmidt, whose IT pedigree includes running Novell and who presently sitting on the board of Google, has famously encapsulated the essence of web2.0: 'don't fight the internet'. I've taken this phrase to sit within an aspect of the theme of this conference the omnipresent internet as god.

Rather than using the internet as a passive platform to be harnessed to fit within the traditional educational paradigm, web2.0 operates to maximise the benefits of interconnectedness and interactivity offered by the internet so we use it as a platform in itself to transform our practice and our experience: using the web, rather than our pc desktop, as our technology platform.

Web2.0 has been characterised as 'a set of economic, social, and technology trends that collectively form the basis for the next generation of the Internet'. 'Web 2.0 is a transformative force that's propelling companies across all industries towards a new way of doing business characterized by harnessing collective intelligence, openness, and network effects. ${ }^{2}$

There are some debates about the 'newness' of this - some, including the 'inventor' of the web Sir Tim Berners-Lee, say that this is simply a description of the worldwide web. Others, including Tim O'Rielly who has promoted the term web2.0, see it as a genuine extension of the initial concept of the web, through the development of a 'set of outcomes enabled by those [initial] web technologies'.

Either way, it is possible to see web 2.0 as having a social and economic foundation, impact and application, facilitated by technology. How then can we harness this in the context of tertiary education? In light of emerging debates about pedagogical foundations of using web2.0 tools, I have taken a cautious approach to using one such technology - blogs - in a first year law subject.

\section{What is a blog?}

A blog is a web log - a 'simple webpage consisting of brief paragraphs of opinion, information, personal diary entries or links arranged chronologically. Most blogs also allow visitors to add a comment. ${ }^{4}$

A blog falls within the web2.0 concept because it provides space for user generated content. You can see from the example in the powerpoint that it is current, brief and topical. The ideas are presented in a range of formats, facilitating interconnectedness and interaction. The ideas are searchable through these links and through metatags embedded within the post.

\footnotetext{
${ }^{1} \mathrm{http}: / /$ radar.oreilly.com/archives/2006/12/web-20-compact.html at 26 April 2009.

2 http://radar.oreilly.com/web2/ at 26 April 2009.

${ }^{3}$ In Paul Anderson 'What is Web 2.0? Ideas, Technologies and Implications for Education' JISC Technology and Standards Watch Febuary 2007, http://www.jisc.ac.uk/publications/publications/twweb2.aspx at 26 April 2009.

${ }^{4}$ Ibid, 7.
} 


\section{Blogging in LA1101}

In my subject, LA1101, we call our blogs blawgs - law blogs. In our blawg task, students are required to make four blawg posts through the semester - an introductory blawg introducing themselves, and three blawgs addressing three different themes: a report of the student's observation of a court proceeding; a critique of legal institutions and processes as reported in a current news article; and an overview of an online journal article or case addressing a set research topic, explaining how the article or case was found and why it is relevant to the topic.

Each post is $300-400$ words. You can see from the powerpoint that our blawgs contain the elements we observed in the 'real mccoy' on the internet...

\section{Designing the Assessment}

The starting point for any assessment has to be the graduate attributes and the learning outcomes for the subject, in the context of the subject content, resources and teaching and learning settings. As with every aspect of subject design, pedagogy (andragogy) must drive design - 'you lead, I'll follow'. This approach to assessment design reflects the principle of alignment. Our first year program in law has extended the principle of alignment beyond a single subject, to the entire first semester suite of subjects.

This subject's learning outcomes focus on development of skills of analysis and critique within the context of Australian legal institutions and processes. These are supported by graduate attributes of speaking and writing clearly, and skill in finding and accessing information using appropriate media and technologies. These attributes are reflected across the first year curriculum, and we have undertaken a lot of research and evaluation of our students' needs in promoting the skills. In first semester in particular, there is a deliberate focus across the first year curriculum on regular lower stakes writing tasks providing regular feedback to students to help improve their writing.

This then generated the idea for this subject, of a variety of low stakes tasks each of which focuses on a different context within which to exercise thinking and writing skills.

The tasks involved in this assessment could however be generated within any medium paper copy, email attachment etc. So when selecting the medium for these tasks we had to ask ourselves what learning outcomes, what graduate attributes we were seeking to promote via not just the thinking skills or content involved, but the medium in which these were to be presented.

In our law school, we are also seeking to embed flexible learning within our course. While this is often seen to manifest in use of technology, we reject the idea of uptake of technology for its own sake, but rather seek to identify opportunities for student centred learning based on sound pedagogical principles. Therefore, in selecting the medium for our tasks we needed to identify how any particular medium would value add to our students' learning experience. 
This is where the concepts of web2.0 come into their own: the ideas behind the assessment task are a true reflection of the ideals of web2.0:

- it seeks to promote a community of learning amongst our students across two campuses through the interconnectedness of the internet as a platform supporting the pedagogy of engagement

- our students can generate their own content within our subject site, over which they have control - promoting learner-centredness

In addition, we present this task as an authentic learning experience, using examples of blawgs on the internet itself as a model for thinking like a lawyer. To ensure equity in student access to this task, we use the standard platform of LearnJCU, and provide detailed instructions on use of the technology involved.

\section{The Perils ...}

One of the biggest challenges we have faced is the load on academic staff of marking, and in communicating feedback with our students. On reflection, and through observing the variety of approaches our team has taken to marking, this challenge is partly a reflection of our own experiences in the web2.0 environment and the constraints within which we have conceptualised this assessment task.

While the task is designed for interconnectedness, it is a somewhat artificial interpretation of the true web tool. First, surveys of our students reveal that $21 \%$ of our respondents in a school-wide survey used blogs. We therefore needed to pitch this tool at a level that was accessible to all students, regardless of their experience with this medium. Accordingly, there is no explicit encouragement for genuine interaction between students through, say the comments function in the blogs. The tasks are prescribed, and students have almost universally stuck to those tasks, not moving outside them.

Likewise, our emphasis on traditional forms of academic and professional written expression albeit presented within the blog, have constrained our thinking into providing an edited, marked up version of the student writing to highlight where improvement is needed.

I started by providing feedback via the online gradebook according to a criteria grid of limited, satisfactory, excellent. Students reported that this held little meaning. My Townsville colleague used this in conjunction with a marked up hard copy of the piece, which was better received. Many of my colleagues report that marking online is difficult for them, and that they prefer hard copy marking. This involves printing all the blogs, marking and handing back.

You can see that this does not accurately represent the interconnectedness of the web platform... 
Part of our initial reluctance to provide feedback via the 'comment' function within the blog itself, lies in the public nature of this forum - we didn't want to expose students' weaknesses to the scrutiny of their peers. Yet reconceptualising feedback in terms of web2.0 principles should draw out additional benefits for students through observing reader reactions to all work posted on the blog.

The second limitation probably lies in our trying to do too much with this task. It could be that using this medium to promote the finest details of professional and academic written literacies (requiring individual marked up feedback) is possibly asking too much. Stripping back our expectations to the immediacy, interconnectivity and interaction of the web2.0 philosophy is more likely to maximise the benefits of the task for students while minimising the perils for the instructors.

\section{The Pitfalls...}

While we had anticipated the perils of marking, the pitfalls, by definition, we were unprepared for.

We had believed the hype associated with gen Y, assuming that our students were technologically literate. Our student surveys since we started the blogs, and our 2008 survey of the entire LLB cohort, reveal otherwise. In fact, as I've mentioned, only $21 \%$ of our student cohort engages in blogging. Even fewer engage in chat rooms or virtual reality sites. Just over half of our students participate in social networking sites.

As a consequence of this, we have had to refine our task as a first step into web2.0 for our students. This task is the first step in a scaffolded approach to development of technological literacy for our student cohort. This has also meant a very high administrative load in responding to student queries about accessing the blog and navigating it. At the moment, this is handled by me as the subject coordinator - however this is not sustainable, and in my view, we require additional resources or a re-thinking of the task to provide adequate support for students. This may be linked to a rethinking of the task more aligned with the contemporary understanding of web2.0 itself.

Access issues have arisen partly because of the way we have structured the blogs, dividing students into blog groups of about 20. The rationale for this was accessibility students report to feeling overwhelmed and nervous about the entire cohort (about 220 students) viewing their work. It was also to facilitate marking by campus, so that we could readily identify students on our own campus by their group allocation, and mark our own students' work.

True web2.0 thinking will not distinguish geographically - interconnectedness is after all global. Again, advancing our thinking to truly embrace the web2.0 concept should help to mitigate student demands for support, through having a fully open forum, rather than small groups of bloggers.

Another issue raised by our students themselves, is their apprehension about having others view their work. This is the flipside of the web2.0 concept: interconnectedness 
represents risk taking which doesn't exist in the context of traditional educational and social paradigm.

\section{And, the pleasures...}

The first real win I think in this assessment, is the level of apparent engagement we have engendered. You'll see from the slide, that after only the introductory post and one substantive task, this group has 396 views, with only 17 members. That's a lot of looks by our students at their own work. Other groups show similar results.

Each year we survey our students on their thoughts on this assessment. Each year they tell us they like reading what other students had to say, and seeing the standard of other students' work. They appreciate the regular feedback, and believe it helps them to improve their writing. The regular tasks also help them keep on top of their subject preparation.

Non-gen $\mathrm{Y}$ students have reported that the tasks have given them confidence in developing technological literacy - it no longer seems daunting to use web technologies.

\section{Conclusion}

In conclusion, our web2.0 assessment tool represents a vehicle for developing our thinking about the context of teaching and learning in an increasingly interconnected and interactive world. It has been challenging to change the paradigm within which we conceptualise subject design and assessment design within the subject. We rely heavily on reflection and evaluation of student feedback to assess the efficacy of our decisions and their impact on our students' learning. This process provides the signposts to help us to make the paradigm shift from what we know to the possibilities of the new digital world. 